\title{
Professor:e - An IMS Standard Based Adaptive E-learning Platform
}

\author{
Cristina Segal and Luminita Dumitriu \\ COMSYS ltd., Al. Marinarilor nr. 2, Mazepa II, Galati, Romania \\ \{Cristina.Segal, Dumitriu.Luminita\}@comsys.ro
}

\begin{abstract}
Recently, the IMS Global Learning Consortium has provided specifications on modeling several aspects related to e-learning. Some of these aspects are separating the actual educational content from the structure of a learning unit, the learner design, the quiz testing, the learning scenario. Learning Simple Sequencing (LSS) is a scenario modeling principle, published in March 2003, meant to help e-learning platform developers to implement educational content delivery modules. Also, an extension of the Content Packaging XML manifest was provided in order to include the new standard.
\end{abstract}

\section{Introduction}

First, we shall review the main contributions of IMS standards to the components of an e-learning platform. Thus, we have to consider several aspects that have been specified by the IMS Global Learning Consortium. The main entities involved in an e-learning platform are the trainers, the trainees and the educational resources, as well as the administration of the entire learning process.

On the management of educational resources, IMS has issued the Content Packaging specification, facilitating the separation between the structure of the learning material and the included information.

The learning process administration is specified by the Enterprise standard that intends to ensure the interoperability between the system and the following components: Human Resources, Student Administration, Training Administration and Library Management.

The student evaluation process is specified at the quiz level, by the Question\&Test Interoperability specification, providing rules for questions and tests representation.

The learning scenario delivery module should manifest the adaptive behavior of the learning system. In order to model the learning scenario (firing rules, constraints, randomization, etc.) IMS issued the Simple Sequencing standard, taking into account the most frequent and simple models leading to a student-adaptive course delivery.

In the following sections, we are presenting the basics of these specifications.

\subsection{Content Packaging Standard}

The fundamental notions of this specification [1] are the package, the manifest and the resource. 
A package designates an usable autonomous content unit. It can be a course, a part of a course or a course collection. As soon as a package is delivered to a run time service, it must allow aggregation/disaggregation into other packages. A package is a stand-alone unit, thus it has to contain all the information required in order to use its contents for learning when unpacked.

A manifest is a XML description of the resources. It contains a variable number of static organizing the instructional resources for presentation.

A resource described in a manifest is a physical content file or a collection of resources described through their own manifests. There can be web pages, documents, text files, media files, data files, as well as external URL-available resources. Each resource is described as a <resource> element in the XML manifest and each associated file as a $<$ file $>$ element within its resource.

From the implementation point of view, a package is defined by:

- a specially named XML file, the top-level manifest;

- all XML control documents it refers along with

- the actual physical files, all grouped together in a logical directory.

The top-level manifest describes the package itself. It contains the following sections:

- meta-data section - an XML element describing the whole manifest;

- organizations section - an XML element describing several organizations of the content within a manifest;

- resources section - an XML element referencing all of the actual resources and media elements needed for a manifest, including meta-data describing the resources, and references to any external files;

- (sub)manifest - one or more optional, logically nested manifests;

- Physical Files - these are the actual media elements, text files, graphics, and other resources as described by the manifest(s). The physical resources may be organized in sub-directories.

Characterizing an instructional content is up to the content developer. If a package is meaningful only as a whole, then it should be described by the top-level manifest only. If a package contains elements that can be used on their own, each one of these elements should be described by a nested (sub)manifest in the top-level one. Thus, disaggregation of content is allowed.

If we consider a package at the curriculum level, the top-level manifest has to comprise all course-level manifests as well as any instructional object of each course.

The benefit of complying to the Content Package standard stands in the interplatform operability of the content as well as in its potential reusability.

\subsection{Enterprise Standard}

The reason behind the Enterprise specification [3] is ensuring the interoperability between the Learning Management System and other systems within an enterprise. The later ones are:

- Human Resource system;

- Student Administration system;

- Training Administration system;

- Library Management system. 
The human resource system is in charge with the skill and competency management, as well as with the selection for learning.

The student administration performs the course catalog management, the class scheduling, class enrollment, attendance tracking, grading, and many other education functions;

The training administration supports course administration, course enrollment, and course completion functions for work force training;

The library management system manages collections of physical and electronic learning objects and manages the access to these materials.

This specification considers that the people-related data from the enterprise system have to be passed to the learning environment, thus creating student profiles. Class/group creation as well as enrolling students and assigning teachers are ensured. Group management can include class scheduling and membership maintenance. Finally, the evaluation and final results recording can occur.

The specification revolves around the following basic concepts: person, group and group membership. A person, in this context, is an individual involved in a learning activity. The required information does not aim to creating a person repository; it only considers learning-relevant information. A group is a collection (generic container) of objects relating to a learning activity; it may contain a class, a tutor, a curriculum, a sub-group, but there are no restrictions on the group structure. The membership is used to include persons and groups to a group. A group may contain any number of persons and groups, while a person or group may be members of any number of groups. The recurrent membership between groups is not allowed.

The XML instance for the Enterprise standard contains any number of persons, groups and membership structures in this order.

\subsection{Learning Simple Sequencing}

The simple sequencing standard defines means of representation for the behavior of a learning experience in such way that a computer-assisted learning system (CAL/CAI) has the ability to decide the sequence of learning activities in a consistent manner. A learning scenario designer can specify the relative order in which learning items will be presented to the learner, as well as the conditions governing selection, delivery and ignoring items during a presentation

The standard [4] comprises rules describing the learning activities branching and browsing through educational content with respect to the outcome of learner-content interaction. While educational content developers have to be able to create and describe the content sequencing, the CAI/CAL systems may hide the details related to the models in the standard to the user. The advantage in committing to this standard consists in interchanging learning activities sequencing between systems.

The ensemble of a system's modules used to fire rules and perform behaviors related to presenting educational content to the learner is called a sequencing engine.

The simple sequencing refers to the limited number of techniques included in the specifications, namely the most frequently used ones. Techniques based on artificial intelligence or schedule-based, or collaborative are neither included nor forbidden by this standard. 
The presentation context is assumed to be a web-based one, but there is no actual restriction. Also, there is no assumption on communication and control interfaces, on the graphical aspect or the functionality of the presentation interface.

Even though the representation extends Content Packaging manifest, implementing the later is not mandatory.

\subsection{Question and Test Interoperability Standard}

This specification [2] aims to enable the exchange of this test and assessment data between Learning management Systems by defining a basic structure for the representation of questions and tests. Thus, the following are provided:

- the definition of questions, choices, feedback/branch and scoring, through standardized attributes (question meta-data along with identification of required and optional elements);

- the interoperability of question collections - definition for packaging and distribution;

- the representation for results reporting;

- the schema for assessment, tracking and presentation;

- APIs for dynamic interface on question retrieval and scoring/assessment engines. The main concepts of the QTI specification are the item, the section and the assessment. The item contains beside the question itself other information related to rendering, scoring and providing feed-back. Different types of questions are considered like multiple choice, fill in the blanks, hot-spot, etc. A section is an assembly of several items or sections, while an assessment is a collection of sections. A participant will only interact with an assessment.

\subsection{Other Related Standards}

Since we have chosen that the learning environment will be web-based deployable, we have included an editing tool allowing the user (author/teacher/student) to compose text, graphics and symbolic expressions. In order to implement these features we have also considered the MathML and SVG standards of representation for graphics and expressions. These standards have an XML-based representation leading to a performance increase due to the dimension reduction of physical content (e.g.: a raster format for a drawing is considerably larger than the vectorial format).

\section{Our Approach}

The e-learning platform we have designed and implemented is based of the mentioned specification. There is no doubt that complying to standards ensures, on one hand, various content integration and on the other grants the soundness of the approach.

Implementing the standards meant instantiating them in a concrete manner. The interpretation we gave to the specifications above is described in this section. 


\subsection{Content Related Aspects}

We have integrated both Content Packaging and QTI standards in order to produce learning content. We have implemented the resource concept enhancing it with an integrated editor of instructional resources. From the QTI standard we have implemented for the moment only multiple choice questions, in order to extend it later with the other possible types.

The author-user can create/manage/modify/delete packages, collection of questions and tests. We have also considered download/upload of packages and question collection to comply with the spirit of the standards.

We have introduced a concept over both standards, the course, which can comprise packages or package sections as well as tests, allowing the teacher/content manager to realize the ensemble of the instructional material required in an e-learning environment.

\subsection{Role Related Aspects}

The roles we have implemented in platform are the following: the student, the teacher, the content manager and the training administrator.

A teacher can assume the content creator/manager role, but it is not mandatory. The student can be a content creator as well, in the context of homework or projects that require editing documents.

The training administration component implements in part the Enterprise standard, meaning enrolling, group management, class creation, etc.

As we can see in Fig. 1, in the left side of the screen, we have a general group of teachers and a general group of students that can register themselves into the platform, for the moment. If a Human Resources System exists, we can consider importing the personal data into the platform, but this is not yet the case.

The training administrator can create a group around an existing course in the platform, attaching students from the general group, as well as trainers from the general teacher group. He can also schedule the classes of course study. From this moment, the student in this group can visualize the course contents, perform the assessments, while the tutor can visualize the grades/scores obtained by the students to whom he is teaching the course.

In what concerns the Library System in the Enterprise standard, we have not considered it since the platform already has Content Packaging and QTI implementation, thus providing its own contents.

\subsection{Student Tracking}

Only student activity is logged for the moment. The information available on student activity is:

- the grades/scores of a student, for all courses he is enrolled to;

- the grades/scores of the students taking on of the courses are visible to the course teacher.

The student access is granted to the course contents to the essay writing section, as well as to his section of the catalogue. 


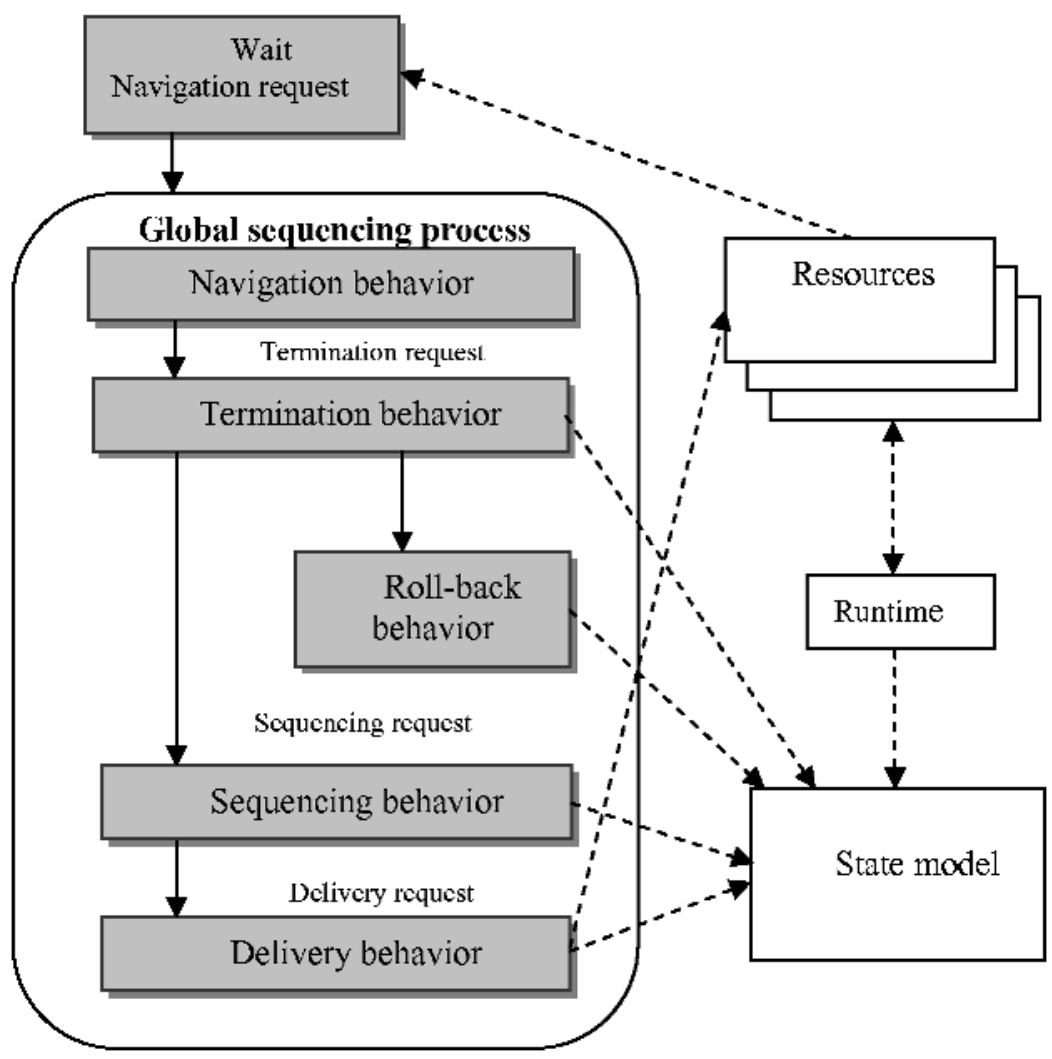

Fig. 1. The sequencing loop from IMS's LSS

\subsection{Sequencing}

For the moment, we have a linear, random-access scenario to the course. We are in the process of implementing the LSS standard, allowing the teacher to adapt the course presentation to the student profile. We are considering the scenario creation process, involving the editing of pre- and post-condition rules, the limit for tentative access to the contents, etc.

The Simple Sequencing approach, proposed by IMS, even called Simple, has a considerable number of modules and behaviors to be harmonized when implementing.

As we can see in Fig. 1, the main interest in the LSS standard, is modeling the navigation behavior, by firing rules that control the number of accesses to the contents, by delivering or skipping sections depending on the student profile.

The student current profile is decided upon completion of a pre-test for each chapter. This way, the teacher can specify thresholds on the mark, or partial mark, a student receives on his pre-test; if student's score is higher than the threshold then a certain section of the course is skipped for presentation.

Another dependence on student profile consists in analyzing the previous instructional path of the student in order to avoid redundancy. This instructional path 
is expressed either through the previous learning activity within the platform or through the instructional past of the student.

An assessment test will consist of questions according to the sections previously presented to the student, meaning that skipped sections will not be materialized as questions in the test.

Whenever the results of an assessment test or a pre-test are poor within a conceptrelated section the student will not be able to advance with the course but will have to consider studying recommended readings relating to that concept and retake the test later.

\section{Conclusion and Future Work}

We have gained a lot of insight knowledge of the e-learning process while implementing the IMS's standards. We are considering for the future to fully implement the QTI standard with all the types of questions. It is also important to build or import the student profiles, as well as to found the Simple Sequencing standard implementation on the profile structure.

\section{References}

1. C. Smythe, T. Anderson, M. McKell, A. Cooper, W. Young and C. Moffatt "IMS Content Packaging Information Model", IMS Global Learning Consortium Inc., http://www.imsglobal.org.

2. C. Smythe, E. Shepherd, "IMS Question \& Test Interoperability:ASI Information Model Specification", IMS Global Learning Consortium Inc., http://www.imsglobal.org.

3. C. Smythe, G. Collier and C. Etesse, "IMS Enterprise Best Practice \& Implementation Guide V1.1", Final Specification, IMS Specification, July 2002, http://www.imsglobal.org.

4. IMS Global Learning Consortium Inc. "IMS Simple Sequencing Information and Behavior Model", http://www.imsglobal.org. 